Genome Biology

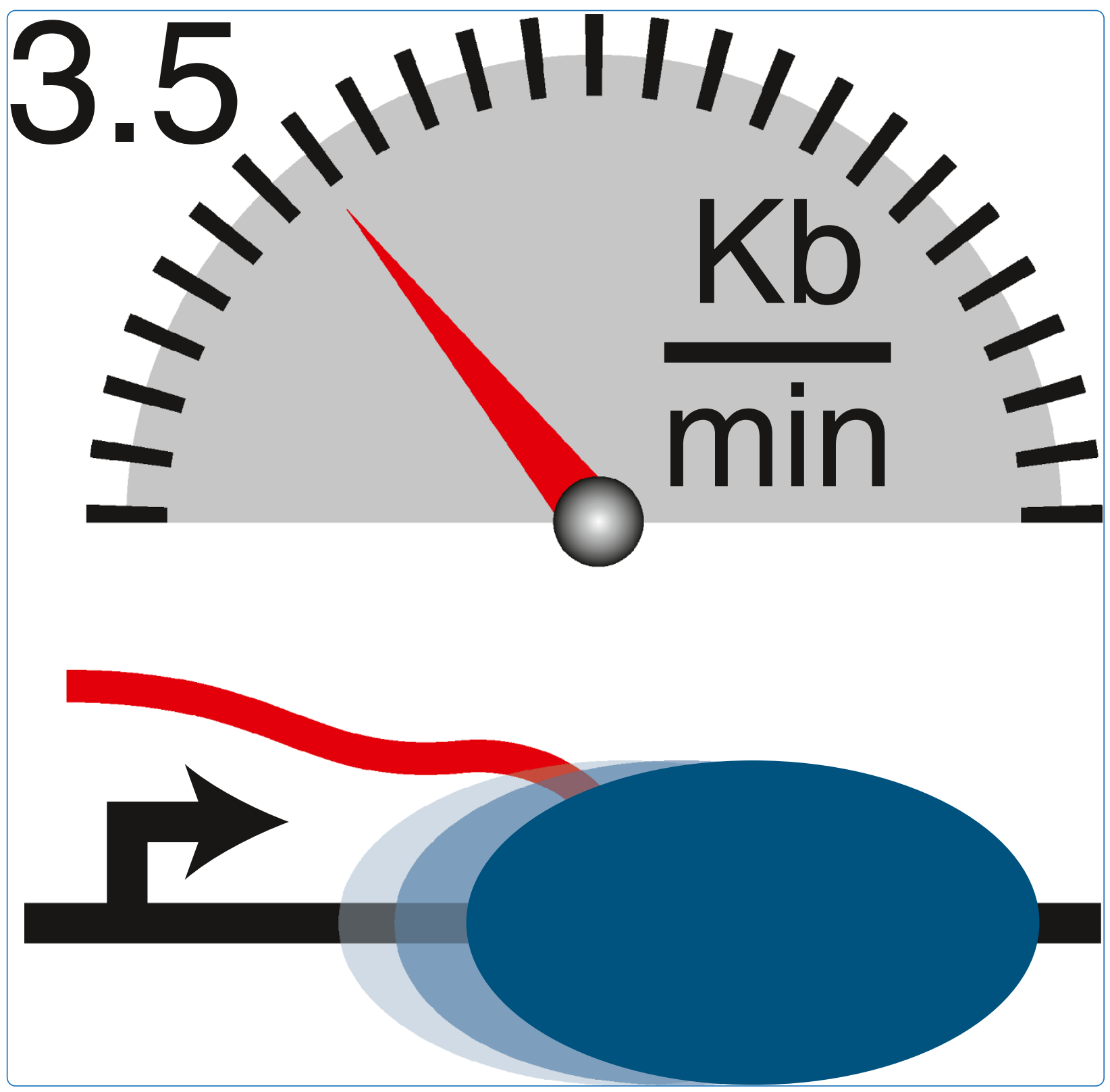

4sUDRB-seq: measuring genomewide transcriptional elongation rates and initiation frequencies within cells

Fuchs et al.

() Biomed Central 


\title{
4sUDRB-seq: measuring genomewide transcriptional elongation rates and initiation frequencies within cells
}

\author{
Gilad Fuchs ${ }^{1 * \dagger}$, Yoav Voichek ${ }^{2 \dagger}$, Sima Benjamin ${ }^{3}$, Shlomit Gilad ${ }^{3}$, Ido Amit ${ }^{4}$ and Moshe Oren ${ }^{1 *}$
}

\begin{abstract}
Although transcriptional elongation by RNA polymerase II is coupled with many RNA-related processes, genomewide elongation rates remain unknown. We describe a method, called 4SUDRB-seq, based on reversible inhibition of transcription elongation coupled with tagging newly transcribed RNA with 4-thiouridine and high throughput sequencing to measure simultaneously with high confidence genome-wide transcription elongation rates in cells. We find that most genes are transcribed at about $3.5 \mathrm{~Kb} / \mathrm{min}$, with elongation rates varying between $2 \mathrm{~Kb} / \mathrm{min}$ and $6 \mathrm{~Kb} / \mathrm{min}$. 4sUDRB-seq can facilitate genomewide exploration of the involvement of specific elongation factors in transcription and the contribution of deregulated transcription elongation to various pathologies.
\end{abstract}

\section{Background}

Gene transcription is a multistep process consisting of recruitment of RNA polymerase II (Pol II) to promoters, transcription initiation, elongation, and termination. In addition to producing RNA polymers based on the DNA template, the Pol II holoenzyme also regulates numerous RNA processing events, including $5^{\prime}$ cap formation, splicing, polyadenylation, and RNA transport [1-7].

While historically most studies focused on understanding how promoter binding and transcription initiation are regulated, recent studies have shown that additional stages of the transcription process are also tightly regulated and are critical for gene activation. These studies have demonstrated that binding of Pol II to gene promoters is not sufficient for productive transcription. Instead, in the majority of genes Pol II is partly 'paused' 20 to $60 \mathrm{nt}$ from the transcription start site (TSS), and several regulated steps are needed in order for Pol II to depart from the TSS and transcribe the rest of the gene [8-15].

The rate of Pol II movement through gene bodies has also been linked to various aspects of co-transcriptional RNA processing. For example, changes in transcription

\footnotetext{
* Correspondence: gilad.fuchs@weizmann.ac.il; moshe.oren@weizmann.ac.il ${ }^{\dagger}$ Equal contributors

'Department of Molecular Cell Biology, Weizmann Institute of Science, Rehovot 76100, Israel

Full list of author information is available at the end of the article
}

elongation rates can affect the outcome of the splicing machinery $[7,16,17]$, and slow elongation by Pol II was shown to enhance the inclusion of specific weak exons [7]; in support of this notion, Pol II was found to accumulate at exons [18-20]. Likewise, the rate of elongation by Pol II has been linked to regulation of alternative polyadenylation [21]; indeed, Pol II accumulates at polyadenylation sites [9].

The importance of steps subsequent to transcriptional initiation is also underscored by the impact of misregulation of these steps on cellular and organismal viability. For example, many of the MLL gene translocation partners, thought to drive aggressive acute leukemia, are part of the super elongation complex (SEC). Leukemiaassociated MLL fusion proteins relocalize the SEC to MLL target genes, bypassing normal transcriptional control and causing aberrant expression of those genes [22]. Similarly, excessive c-Myc was suggested to augment gene expression by increasing the release of paused Pol II and thus facilitating active elongation, thereby alleviating rate-limiting constraints on tumor cell growth and proliferation [23]. Furthermore, viruses utilize or modify transcription elongation-related processes to their benefit. For example, the influenza A NS1 protein comprises a histone-like sequence that can target the PAF1 elongation complex, enabling selective modulation of host cell gene expression and contributing to suppression of the 
antiviral response [24]. In addition, during active HIV-1 infection, viral transactivator of transcription (Tat) recruits the SEC to the HIV-1 long terminal repeat (LTR) to activate expression of the provirus in host cells [25-27]. Thus, better elucidation of the regulation of transcriptional elongation could contribute to the understanding of molecular mechanisms of disease, and even suggest novel therapeutic approaches.

Despite the documented links between elongation rates and numerous RNA-related functions, the actual elongation rates within cells remain under debate, with reported values ranging from 1 kilobase per minute $(\mathrm{Kb} / \mathrm{min})$ to $6 \mathrm{~Kb} / \mathrm{min}$ [28]. In most cases, elongation rates of only a few genes at a time were measured; one study, utilizing Global Run-On sequencing (GRO-seq), was able to determine the rates for approximately 166 long genes upregulated in response to short treatment with physiological, non-toxic inducers [29]. Since the elongation rates of individual genes can be altered in a stimulus-dependent manner [29], it is important to measure the elongation rates of non-stimulated genes in order to derive more general understanding of the relationship between basal transcription elongation rates and steady state RNA processing and gene expression. Moreover, assessment of a large number of genes may provide more definitive information on the factors that affect transcription elongation rates. Here we describe a method that enables to easily measure simultaneously, in a single experiment, the steady state elongation rates of thousands of genes within live cells.

\section{Results and discussion}

Adaptation of the DRB assay for measuring elongation rates in short time scales by RNA sequencing

Previously, Singh and Padgett [30] employed 5,6-dichlorobenzimidazole 1- $\beta$-d-ribofuranoside (DRB), which reversibly blocks transcription in vivo, combined with quantitative reverse transcriptase-PCR (qRT-PCR), to assess the elongation rates of several long human genes. We sought to adapt this method for simultaneous determination of the elongation rates of all DRB-sensitive genes. Since the median length of a human gene is approximately $24 \mathrm{~Kb}$ and typical elongation rates are estimated at a few $\mathrm{Kb} / \mathrm{min}$, measurements were performed 4 and $8 \mathrm{~min}$ after DRB removal. Pre-mRNA levels were quantified by qRT-PCR, employing primers specific for intronic sequences. Analysis of pre-mRNA levels in HeLa cells incubated with DRB for $3 \mathrm{~h}$ revealed that transcription of a proximal region of the OPA1 gene, located $2 \mathrm{~Kb}$ downstream to the TSS, was recovered already within $4 \mathrm{~min}$ of DRB removal (Figure 1A). In contrast, full transcriptional recovery of a distal region of the same gene, located $10 \mathrm{~Kb}$ downstream to the TSS, occurred only 8 min after drug release. A similar trend was observed for TTC17 (Figure 1A) and several other genes (data not shown). Hence, the DRB protocol can capture the progress of Pol II also in average-sized genes.

In order to obtain genomewide information on elongation rates, this protocol had to be adapted to RNA-seq. Nascent RNA constitutes only a very small fraction of the total RNA within cells; therefore, it is necessary to enrich it prior to sequencing. In principle, such enrichment is achievable by GRO-seq, which is becoming increasingly popular [29,31-33]. However, GRO-seq requires prior isolation of nuclei, which takes a relatively long time; this might introduce biases in short time scale measurements. We therefore added to the protocol a step of labeling nascent RNA in vivo with 4-thiouridine (4sU), which can be incorporated into intact cells $[34,35]$, followed by specific purification of $4 \mathrm{sU}$ containing RNA.

To test whether short $4 \mathrm{sU}$ labeling can indeed enrich for nascent RNA, HeLa cells were treated with $4 \mathrm{sU}$ for 8 min. 4sU-tagged RNA was biotinylated in vitro, purified on streptavidin beads and subjected to qRT-PCR analysis. Pre-mRNA/mature RNA ratios were calculated for OPA1 and TTC17 both in the 4sU-enriched fraction and in the total (non-enriched) RNA. Similar to a previous report [35], the short $4 \mathrm{sU}$ labeling yielded an approximately 20- to 35-fold enrichment of nascent RNA of these genes relative to the total RNA population (Figure 1B).

We next set out to determine the rate of movement of RNA Pol II on individual genes. To that end, we monitored the changes in nascent RNA abundance throughout those genes at different time points after resumption of RNA synthesis following DRB removal. HeLa cells were treated with DRB for 3 hours and harvested either 4 or 8 min following DRB removal. In both cases, the cells were pulsed with $4 \mathrm{sU}$ for the last $8 \mathrm{~min}$ before being harvested (Additional file 1). Cells were harvested in QIAzol lysis buffer directly on the culture dish. Next, 4sU-tagged nascent RNA from each sample was biotinylated and collected on streptavidin beads. This experimental procedure was performed separately in two biological repeats for the $4 \mathrm{~min}$ samples and four biological repeats for the $8 \mathrm{~min}$ samples. Finally, the RNA was subjected to deep sequencing (see Materials and Methods). The entire protocol, schematically illustrated in Figure $1 C$, was termed 4sUDRB-seq.

\section{Genomewide analysis of transcription elongation rates by 4sUDRB-seq}

Given the high correlation between biological repeats (Additional file 2), we averaged the 4sUDRB-seq signals of the 4 and 8 min samples from the biological repeats over all genes longer than $20 \mathrm{~Kb}$. We observed a distinct 

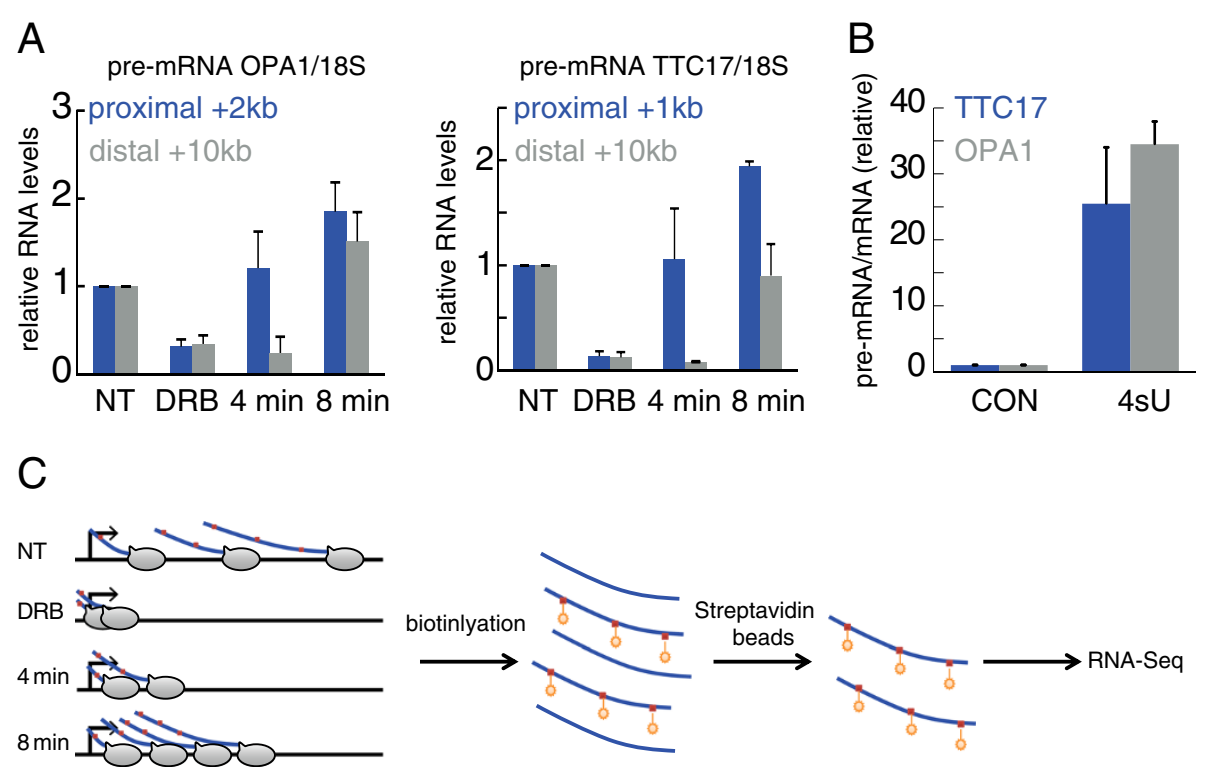

Figure 1 Adaptation of the DRB assay for measuring elongation rates in short time scales by RNA sequencing. (A) Analysis of OPA1 and TTC17 pre-RNA in HeLa cells, immediately after $3 \mathrm{~h}$ DRB treatment (DRB) and at the indicated time points after DRB removal. NT = non-treated. Pre-mRNA levels were determined by qRT-PCR, employing intronic primers. All values were normalized to 185 RNA in the same sample. Levels in non treated cells were set as 1. Bars indicate averages of three independent experiments; error bars represent standard deviation. (B) Relative pre-mRNA/mRNA ratios in total RNA (CON) and 4sU-enriched RNA (4sU). HeLa cells were labeled for 8 min with 4sU (1mM). RNA was isolated, biotinylated, and enriched on streptavidin beads. QRT-PCR analysis of OPA1 and TTC17 pre-mRNA and mRNA was performed on the enriched RNA, as well as on total (non-enriched) RNA. The pre-mRNA/mRNA ratio for each gene in the CON sample was arbitrarily defined as 1.0. (C) Scheme of the timing of DRB and $4 \mathrm{sU}$ treatments in the different conditions. NT = non-treated, DRB $=3 \mathrm{~h}$ of DRB only, $4 \mathrm{~min}=3 \mathrm{~h}$ of DRB and harvesting $4 \mathrm{~min}$ after DRB removal, $8 \mathrm{~min}=3 \mathrm{~h}$ of DRB and harvesting $8 \mathrm{~min}$ after DRB removal. Red squares $=4 \mathrm{~s} \mathrm{U}$, orange stars $=$ biotin. Following the indicated treatments, isolated $4 \mathrm{~s} U$-labeled RNA was biotinylated and purified with magnetic streptavidin beads as described in [42] and subjected to high throughput sequencing.

wave of transcription progression (Figure 2A). As expected, the nascent RNA reads in the 8 min sample extended much beyond the $4 \mathrm{~min}$ sample, relative to the TSS (marked as 0). Of note, the average distance traversed by Pol II between 4 and 8 minutes appears substantially longer than that attained in the first 4 min; this might imply either a slower elongation rate in the region immediately adjacent to the TSS followed by subsequent acceleration, or a short delay in resumption of transcription following DRB removal. A similar pattern was revealed by examination of single gene reads, showing a clear advance of nascent RNA synthesis between the 4 min and 8 min time points (Figure 2B, C).

The density of reads decreases gradually towards the advancing front of the transcription wave (Figure 2B, C). Hence, the exact border of the advancing transcription wave is not sharp and is hard to define. Furthermore, DRB does not enforce a complete transcription arrest, as evident by the presence of scattered low abundance reads throughout gene bodies (Figure 2B, C). Therefore, we developed an algorithm that determines the front border of the elongation wave for each gene at a given time point, taking into consideration the above observations. To avoid biases due to sequence properties, the
pre-DRB release pattern was used for correction. Only genes with a primary transcript longer than $25 \mathrm{~Kb}$ were considered.

To determine the front end of the elongation wave for each transcript at 4 and 8 min after DRB release, we performed two sequential steps. First, we attempted to define the approximate position of the front end ('rough' estimate) by identifying the 5 '-most point within the gene where the number of normalized reads is not any higher than at the 0 time point (Figure $3 \mathrm{~A}$ ). In the second step, we refined this estimate using the shape of the pattern. Basically, the algorithm looks at the derivative of the pattern while correcting for the background. As the abundance of reads decreases towards the front boundary of the elongation wave, the pattern derivative should become negative; however, at the exact location of the boundary it should become 0 , as it is not affected anymore by transcription initiation due to DRB release (Figure 3A). Hence, the first position upstream to the region identified by the 'rough' estimate where the derivative became 0 was defined as the wave end point.

In some cases, the algorithm identified a clear front boundary for a particular gene in some of the experiments, but failed to do so in one or more of the other 


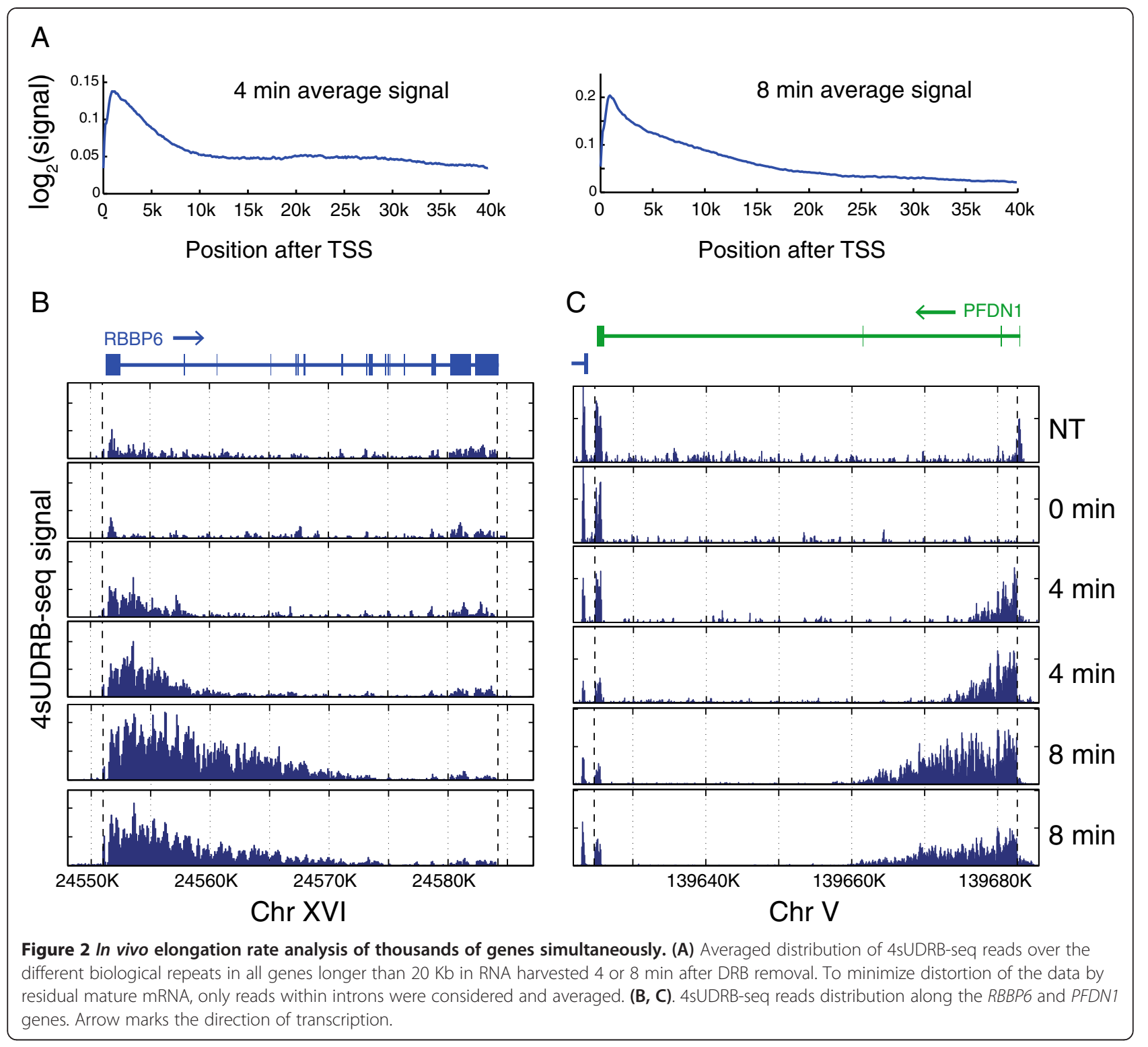

experiments (data not shown). Consequently, we calculated elongation rates only for genes that yielded distinct boundaries in at least two independent repeats of a given time point. To assess the reliability of our measurements, the confidence level of the elongation rate for each gene was calculated (Materials and Methods). As seen in Additional file 3, for most of the genes the confidence level was better than $\pm 0.5 \mathrm{~Kb} / \mathrm{min}$. For subsequent analysis, only genes with confidence levels better than $\pm 0.5 \mathrm{~Kb} / \mathrm{min}$ were considered (Additional file 3 ). In addition, the estimated delay time, namely the time it takes transcription to reinitiate effectively following DRB removal, was also calculated; this was defined as the time interval missing in order for the distance between the TSS and the $4 \mathrm{~min}$ boundary to be equal to the distance between the 4 and $8 \mathrm{~min}$ boundaries, given the calculated elongation rate. This calculation is based on the assumption that the elongation rate is uniform throughout the gene. However, it remains formally possible that the rate is slower at the beginning of the gene, in which case the calculated delay times may represent overestimates.

Overall, we could measure with high confidence the elongation rates of 1,577 genes. The full list of elongation rates generated by this analysis is presented in Additional file 4. Examples of elongation rate measurements for several representative genes are shown in Figure 3B. As also seen in Figure 3C, while most genes are transcribed at a rate of approximately $3.5 \mathrm{~Kb} / \mathrm{min}$, actual transcription elongation rates vary between $2 \mathrm{~Kb} / \mathrm{min}$ and $6 \mathrm{~Kb} / \mathrm{min}$. Overall, these rates are in the same range as those determined previously for a small number of specific genes $[28,30]$. 

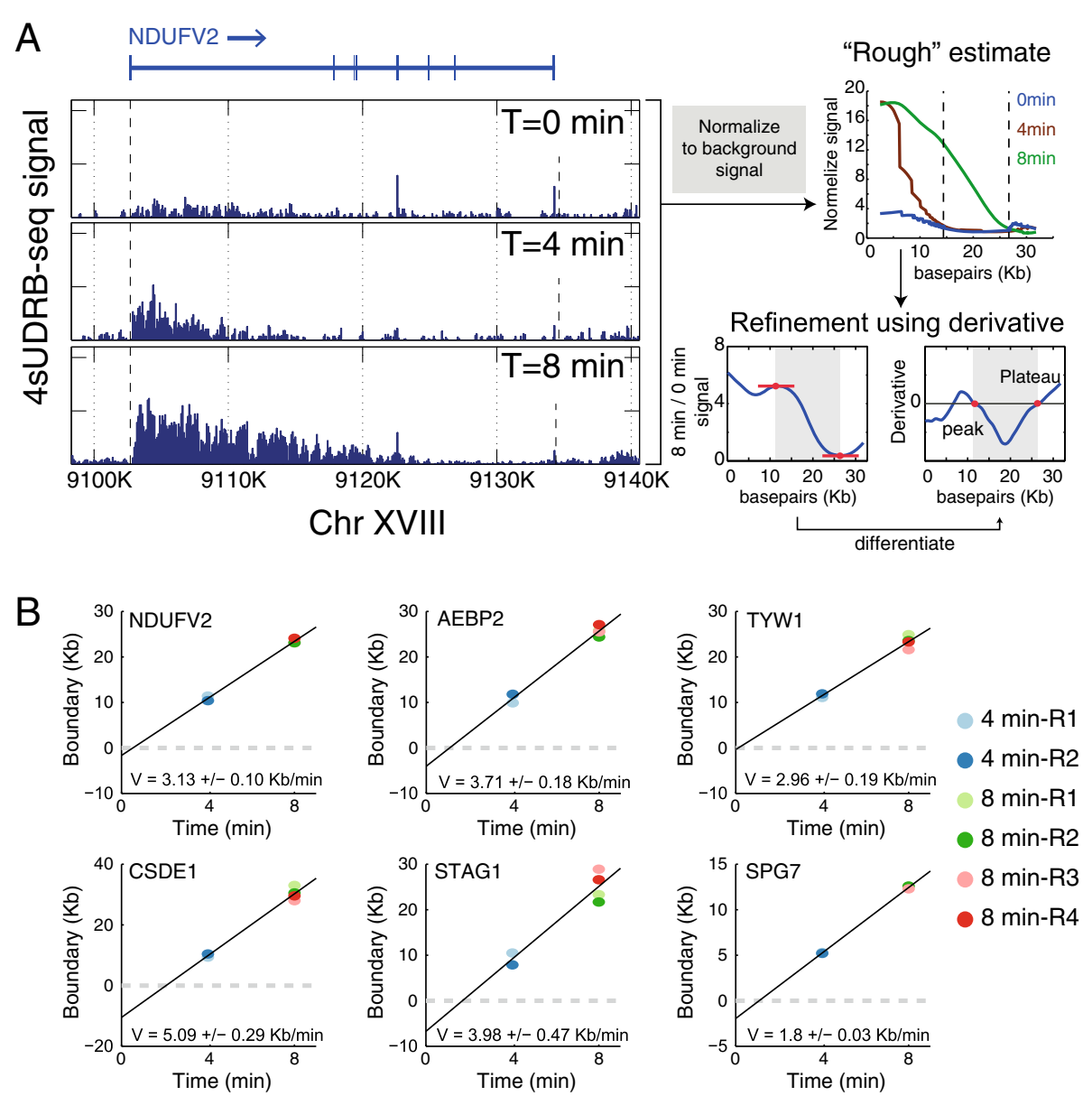

C
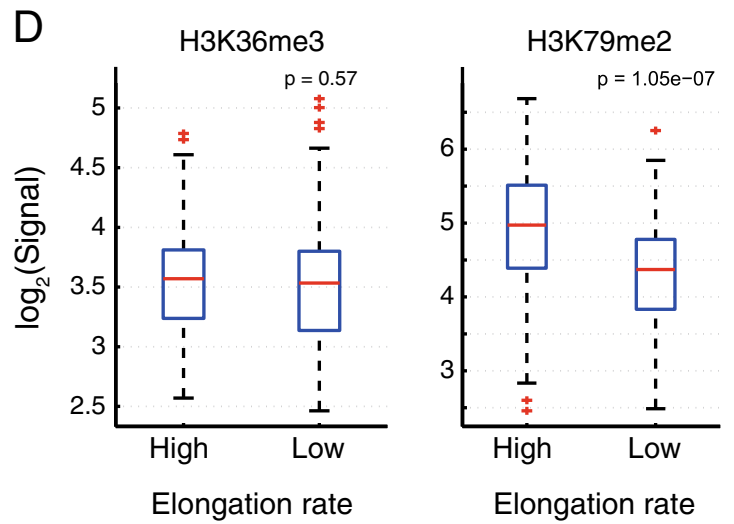

Figure 3 In vivo genomewide measurement of transcription elongation rates. (A) Schematic representation of the algorithm used to infer elongation rates, exemplified for the NDUFV2 gene. Left panel: 4sUDRB-seq data for 0/4/8 min after DRB release (similar to Figure 2B). Each signal was then corrected for the inferred background signal (right top panel), identifying for the 4 and 8 min samples the first position downstream to the TSS where the corrected signal is similar to that of the 0 time point; this location is designated by a vertical dashed line. To refine the boundary identification, we evaluated the background-corrected signals of the 4 and 8 min time points divided by the 0 time point signals and their derivatives. Refinement of the boundary position was then performed by monitoring the decreasing signal area in the vicinity of the rough boundary estimate, and determining the location between the closest peak and the point where the signal plateaus. (B) Linear fitting was performed on the averaged 4 and 8 min elongation boundaries as a function of time for the indicated genes. Elongation rates were defined by extracting the slope value of the linear fit $(\mathrm{V})$. Confidence interval is indicated for each gene. (C) Distribution of measured elongation rates of all informative DRB-sensitive genes. (D) Box-whisker plot of log2 transformed H3K36me3 and H3K79me2 levels in genes that overlap with each modification in the $25 \%$ of genes with the highest calculated elongation rate and the $25 \%$ of genes with the lowest elongation rate. H3K $79 \mathrm{me} 2$ is significantly higher in fast elongating genes (t-test, $P=1 \mathrm{e}-7$ ). All data were adapted from ENCODE. 
To validate the rates deduced by 4 sUDRB-seq, two genes with different calculated elongation rates (RBM33 $=4.53 \mathrm{~Kb} / \mathrm{min}, C L C N 3=2 \mathrm{~Kb} / \mathrm{min}$ ) were examined at higher temporal resolution, starting at $2 \mathrm{~min}$ after DRB release. Pre-mRNA was quantified by qRT-PCR with intron-derived primers. Reassuringly, the elongation rates determined by qRT-PCR $(R B M 33=5 \mathrm{~Kb} / \mathrm{min}, C L C N 3=$ $2.7 \mathrm{~Kb} / \mathrm{min}$ ) were in reasonably good agreement with the rates determined by 4 sUDRB-seq (Additional file 5A); the small differences might be due to the lower spatial resolution of qRT-PCR as compared to RNA-seq. Similar results were observed by measuring the elongation rates of those genes with different sets of primers (Additional file 5B).

Histone post-translational modifications (PTMs) were suggested to alter transcriptional elongation rates [36-38]. Hence, we asked whether the variance in elongation rates correlates with differences in a particular PTM. Interestingly $48 \%$ of genes in the upper quartile of genes with the highest elongation rate were found to be enriched in H3K79me2 within their gene body, compared to only $19 \%$ of genes from the slowest elongation rate quartile (Fisher's exact test, $P=5.5 \mathrm{e}-18$; data not shown). In contrast, no significant enrichment was observed for H3K36me3 (Fisher's exact test, $P=0.99$ ). Further analysis of genes that have H3K79me2 or H3K36me3 in their gene body confirmed that H3K79me2 is significantly higher in the fast elongation rate quartile (t-test, $P=1 \mathrm{e}-7$ ), while $\mathrm{H} 3 \mathrm{~K} 36 \mathrm{me} 3$ is relatively similar in both quartiles (t-test, $P=0.57$ ) (Figure 3D). Since the average expression level was similar in both quartiles (t-test, $P=0.43$, data not shown), the difference in H3K79me2 cannot simply be due to differences in expression. Further experiments will be required in order to determine whether H3K79me2 accelerates elongation or the high levels of this PTM are merely a consequence of the faster elongation. The fact that we did not detect a correlation between H3K36me3 and elongation rates is surprising, since such correlation was recently observed in response to a specific stimulus [29]. This might suggest that elongation during induced transcription utilizes a different set of histone PTMs than those associated with constitutive basal transcription.

\section{Calculation of genomewide relative transcription initiation frequencies by 4 sUDRB-seq}

The 4sUDRB-seq reads are not uniformly distributed throughout gene bodies, decreasing gradually towards the 3 ' end of the gene (see Figures 2 and $3 \mathrm{~A}$ ). The slope of the decreasing signal is dictated by the combined impact of the transcription initiation frequency and the elongation rate [39], "transcription initiation frequency" being defined here as the average frequency at which Pol II molecules transition from an initiation mode into an elongation mode and start moving into the gene body (Figure 4A) [40]. Thus, a greater number of such events occurring within a defined time window will result in a steeper slope (Figure 4A, Higher Frequency), whereas a faster elongation rate will tend to flatten the slope (Figure 4A, Faster Elongation). Hence, by measuring the slopes and elongation rates for each gene, the initiation frequencies can be estimated (Figure 4A, B). Using this approach, we calculated the relative initiation frequencies for all DRB-sensitive genes (Additional file 6). To exclude the possibility that our calculation is biased by an excessively high signal in the proximity of the TSS, owing to Pol II pausing, relative initiation frequencies were recalculated for all genes after excluding the 2 first $2 \mathrm{~Kb}$ immediately downstream to the TSS. As seen in Additional file 7, this did not affect significantly the deduced initiation frequencies. Next, we compared the

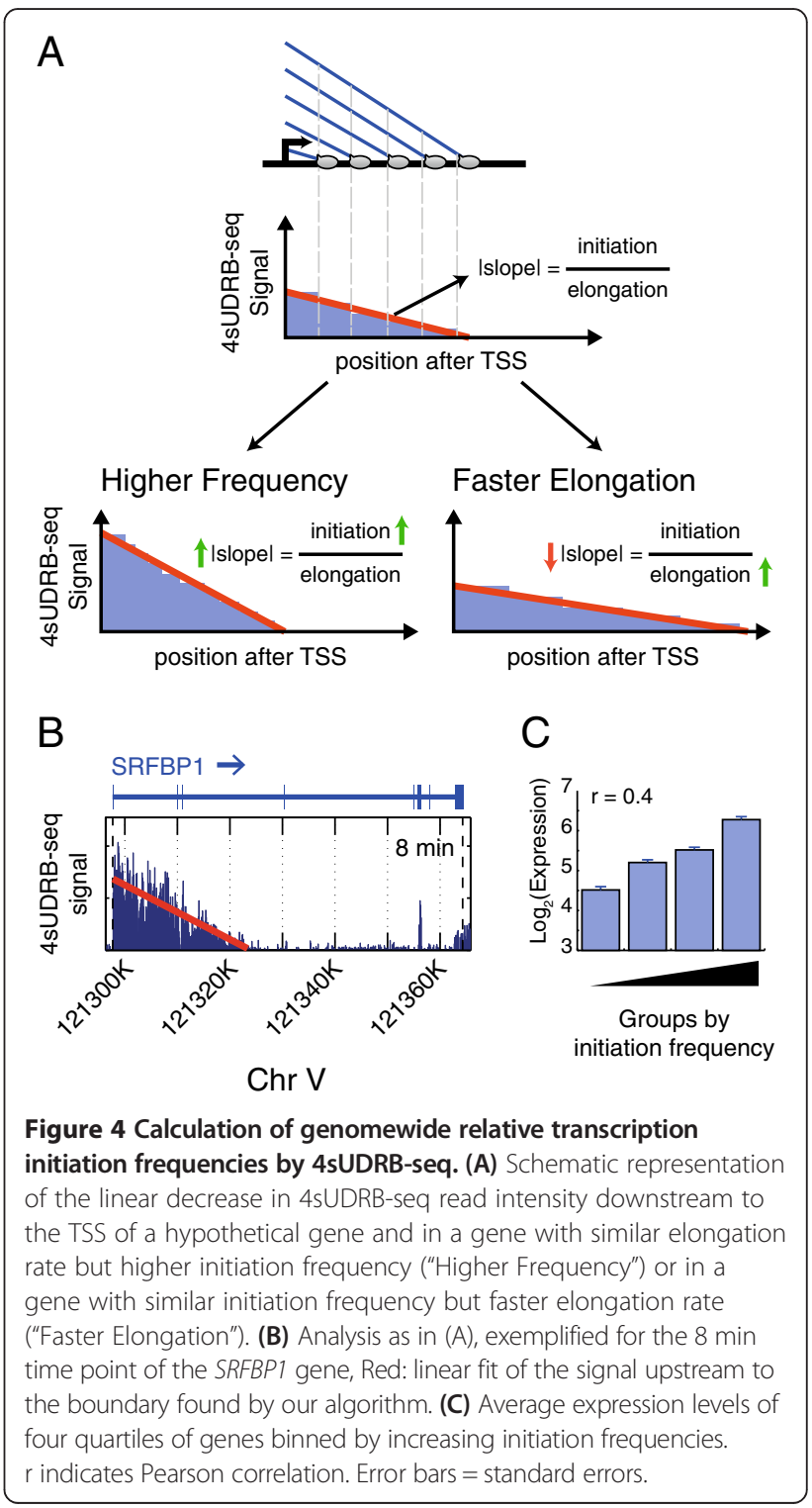


calculated relative initiation frequencies to gene expression data in HeLa S3 cells (ENCODE). As seen in Figure 4C, a positive correlation was found between initiation frequencies and expression levels. Since gene expression levels are mostly determined by initiation frequencies [40], this correlation supports the reliability of the relative initiation frequencies deduced by 4 sUDRB-seq.

\section{Conclusions}

We report here that, by combining the reversible inhibitor $\mathrm{DRB}$ and $4 \mathrm{sU}$ tagging, transcription elongation rates and relative initiation frequencies within intact cells can be relatively easily measured on a genomewide scale. This method can be utilized in order to compare elongation rates between different regions within genes, different tissues, or different biological contexts such as differentiation and transformation. Moreover, by coupling 4sUDRB-seq with depletion of specific transcription-regulatory factors, one should be able to discriminate between factors that impact gene expression by modulating transcription initiation frequencies and those that affect the elongation step.

Note: while this manuscript was under revision, an essentially similar method was described by Veloso et al. [41].

\section{Materials and methods}

\section{Cell culture and treatments}

Human cervical carcinoma HeLa cells were grown in DMEM with $10 \%$ bovine serum supplemented with antibiotics and maintained at $37^{\circ} \mathrm{C} .5,6$-dichlorobenzimidazole 1- $\beta$-d-ribofuranoside (DRB) was purchased from Sigma (D1916), and used at a final concentration of $100 \mu$ M. 4-thiouridine (4sU) was purchased from Sigma (T4509) and used at a final concentration of $1 \mathrm{mM}$. In two of the NT, 0 and 8 min samples, cells were transfected with non-targeting siRNA (Dharmacon) $48 \mathrm{~h}$ before addition of DRB, employing the Dharmafect 1 reagent according to the manufacturer's instructions.

\section{RNA purification and quantitative RT-PCR}

For quantitative reverse transcriptase PCR (qRT-PCR) analysis, total RNA was extracted with the miRNeasy kit (Qiagen). Two microgram of each RNA sample was reverse transcribed with Moloney murine leukemia virus reverse transcriptase (Promega, Madison, WI, USA) and random hexamer primers (Applied Biosystems). Realtime PCR was done in a StepOne real-time PCR instrument (Applied Biosystems, Foster City, CA, USA) with Syber Green PCR supermix (Invitrogen).

\section{Biotinylation and purification of 4sU labeled RNA}

Biotinylation and purification of $4 \mathrm{sU}$ labeled RNA was done as described in [42], with minor changes. A total of 100 to $180 \mu \mathrm{g}$ RNA was used for the biotinylation reaction. 4sU-labeled RNA was biotinylated using EZ-Link
Biotin-HPDP (Pierce), dissolved in dimethylformamide (DMF) at a concentration of $1 \mathrm{mg} / \mathrm{mL}$ and stored at $-80^{\circ} \mathrm{C}$. Biotinylation was done in labeling buffer $(10 \mathrm{mM}$ Tris pH 7.4, $1 \mathrm{mM}$ EDTA) and $0.2 \mathrm{mg} / \mathrm{mL}$ Biotin-HPDP for $2 \mathrm{~h}$ with rotation at $25^{\circ} \mathrm{C}$. Unbound Biotin-HPDP was removed by chloroform/isoamylalcohol (24:1) extraction using MaXtract (high density) tubes (Qiagen). RNA was precipitated at $20,000 \mathrm{~g}$ for $20 \mathrm{~min}$ at $4^{\circ} \mathrm{C}$ with a 1:10 volume of $5 \mathrm{M} \mathrm{NaCl}$ and an equal volume of isopropanol. The pellet was washed with an equal volume of $75 \%$ ethanol and precipitated again at 20,000 $g$ for $10 \mathrm{~min}$ at $4^{\circ} \mathrm{C}$. The pellet was left to dry followed by resuspension in $100 \mu \mathrm{L}$ RNase-free water. Biotinylated RNA was captured using Dynabeads MyOne Streptavidin T1 beads (Invitrogen). Biotinylated RNA was incubated with $100 \mu \mathrm{L}$ Dynabeads with rotation for $15 \mathrm{~min}$ at $25^{\circ} \mathrm{C}$. Beads were magnetically fixed and washed with $1 \times$ Dynabeads washing buffer. RNA-4sU was eluted with $100 \mu \mathrm{L}$ of freshly prepared $100 \mathrm{mM}$ dithiothreitol (DTT), and cleaned on RNeasy MinElute Spin columns (Qiagen).

\section{Preparation and sequencing of $4 \mathrm{~s} U$ labeled RNA}

cDNA libraries were prepared with Illumina TruSeq RNA sample preparation v2 kit according to the manufacturer's instructions but without the polyA isolation stage. Of note, strand specificity is not achieved with this kit. Random hexamers were used for reverse transcription. Libraries were pooled and sequenced on an Illumina HiSeq 2500 system using the paired-end 50 mode.

\section{Read mapping}

All reads were aligned to the human reference genome (hg19, GRCh37) using the bowtie 2 aligner with default parameters (only setting $-\mathrm{N} 1$ ) [43]. We considered only reads mapped uniquely to the genome and not to chrUn_gl000220 (rRNA) for further analysis. For each experiment we constructed a genome-wide profile of the signal per disjoint $100 \mathrm{bp}$ adjacent bins. The sum of each experiment was normalized to be $10^{6}$.

\section{Gene annotations}

All gene annotations were taken from the hg19 RefGene tab. of the UCSC browser.

\section{Boundary detection algorithm}

Only genes longer than $25 \mathrm{~Kb}$ and with at least 20 intron bins with positive numerical values were included in the boundary detection algorithm. In addition, in cases where a gene has multiple variants, we consider only the longest one.

\section{'Rough estimate'}

For each of the filtered genes from the different $4 \mathrm{~min}$ or $8 \mathrm{~min}$ samples we extracted only the profile data of 
introns, in order to avoid the confounding effects of reads derived from contaminating mature mRNA. In order to increase the reliability of the signal used for normalization, and to reduce the effects of variability between the 0 min samples due to low read numbers, the profile of the 0 time point was created by averaging four different biological repeats of the 0 min samples. Next, the intron data were smoothed using cubic spline (smoothing parameter $-10^{-5}$ ).

To normalize each gene in each experiment $(0,4$, and $8 \mathrm{~min}$ ) by the background signal, we performed the following procedure. First, for each bin of the gene we estimated the background signal downstream to this bin up to the 3' end of the gene. This background read distribution was built out of a negative binomial signal. To estimate the average of the background distribution we took the most abundant value. The most abundant value was identified by building a histogram of the data and selecting the bin with the highest count. In cases where we had fewer than 10 values downstream to the last point with positive signals we used the background estimate of the upstream bin.

We next compared the 4 and 8 min normalized profiles to the 0 time point. For each of the 4 and 8 min samples, we chose the first bin downstream the TSS $+2.5 \mathrm{~Kb}$ (defined arbitrarily) where the sample's signal is not higher than the 0 time point at the same position (up to 10\%). This bin was defined as the 'rough estimate' of the boundary. If the first position (TSS $+2.5 \mathrm{~Kb}$ ) was identified or no position was identified no boundary was determined.

\section{Refinement using profile derivative}

For each gene we took the profile of the introns and as in the 'rough estimate', we used cubic spline with the same parameter. Before smoothing the 0 time point bins, we added the value 0.01 to each bin (according to the 'Rule of Succession') in order to prevent cases where data are normalized to 0 time points and results in dividing by 0 . Then we divided the 4 and 8 min gene profiles by the 0 time point profile and obtained the derivative by subtracting each adjacent bin.

We looked for the local minimums of the derivative using the N.Yoder peak finder (mathworks.com) upstream the 'rough estimate' boundary; we considered only derivatives smaller than -0.01 . We took the most downstream minimum relative to the 'rough estimate' boundary. Next, we identified the most downstream bin relative to the minimum (up to $2 \mathrm{~Kb}$ downstream to the 'rough estimate') where the derivative was close to 0 $(>=-0.002)$ and defined it as the 'refined estimate'. If no such bin was found we adhered to the 'rough' estimate.

\section{Calculating elongation rate and rate confidence interval}

In order to calculate the elongation rates, only genes with for which clear elongation boundaries were identified by the algorithm in both of the 4 min samples and in at least three of the four 8 min samples were included. Next, genes in which the average of the $8 \mathrm{~min}$ boundaries was lower than that of the $4 \mathrm{~min}$ boundaries were excluded. Since we had four biological repeats for the 8 min samples, we used modified Thompson tau outlier method in order to eliminate a maximum of one outlier. Next, the elongation rate was calculated by linearly fitting the averaged 4 and $8 \mathrm{~min}$ boundaries as a function of time. The slope of the linear fit was defined as the elongation rate. Next, the $50 \%$ confidence interval of the slope was defined as the confidence interval of the elongation rate. Only genes with confidence intervals better than $0.5 \mathrm{~Kb} / \mathrm{min}$ were retained. The slope was also used to define the delay time, which was defined as being equal to the slope intersection with the time axis. The elongation rate was determined only for genes with delay times between -1 and $4 \mathrm{~min}$.

\section{Processing of ENCODE data}

H3K36me3/H3K79me2 data of Hela-S3 were derived from the ENCODE project [44]. Peak calling for H3K36me3 and H3K79me2 was done as follows: for each gene the $20 \mathrm{~Kb}$ downstream to the TSS were binned into $100 \mathrm{bp}$ bins, and peak calling was done using N.Yoder peak finder (mathworks.com). Only genes with at least one peak were selected. Next, in order to get an enrichment level for each gene we averaged for each modification the signal for all bins.

RNA-seq data of Hela-S3 were also from the ENCODE project (Caltech RnaSeq Helas3 200SigRep1V4) [44]. Expression per gene was the average for the signal on all exons.

\section{Calculating relative initiation frequency}

For a constant initiation frequency $I$ and a constant RNA polymerase velocity $V$, the relative amount of polymerases that have moved downstream to location $x$ in the gene within $T$ minutes ( $T=8$ in our case) from DRB removal will be (with delay time $D$ ):

$$
\operatorname{Signal}(x)=I\left(T-D-\frac{x}{V}\right)=I(T-D)-x\left(\frac{I}{V}\right)
$$

To infer the initiation frequency we calculated the best linear fit of the intron signal as a function of $\mathrm{Kb}$ downstream to the TSS: $a x+b$. If we denote by B the 8 minutes boundary, we get:

$$
a B+b \approx 0(\text { Not exactly zero due to noise })
$$

Adding the previous equality we get:

$$
I \approx \frac{b V}{B}
$$

The intron signal was derived from each 8 min sample, divided by the averaged signal from the 0 min samples 
plus 1 (as pseudocounts to prevent dividing by 0 ). This calculation was performed separately for all four biological repeats and outliers were excluded using the modified Thompson Tau outlier method. Relative initiation frequencies were determined by averaging the rates from the biological repeats.

\section{Primers}

Primers used in this study: OPA1 mRNA F $5^{\prime}$-TT TTTACCTCAGGTTCTCCGGA, OPA1 mRNA R 5'CA CGATCTGTTGCTCTAAACGC; OPA1+2 Kb F 5' ACC ATGGATGCCATTGAGTCA, OPA1+2 Kb R 5'TGTGC CATCACCAGGAGACAT; OPA1+10 Kb F 5'TCTGTT CCATGATGAGCTGTGG, OPA1+10 Kb R 5'CCTGGT CCTTCCTGAATCTTTG; TTC17 mRNA F 5'ATCAA AGCCAAGGTGCCCT, TTC17 mRNA R 5'GGACTGA TGTCTTTGCTCTCCA; TTC17+1 Kb F 5' TCAGAGG CGAGACTGCTTTTC, TTC17+1 Kb R 5'GCATTTAC AAAACGCAGGCA; TTC17+10 Kb F 5' TCCAGCCTC AGACACCACTTT, TTC17+10 Kb R 5' TGGTTTGA AGAACATCCCGAG; RBM33+5 Kb F 5'TTCCACATC TTCCTGGCACA, RBM33+5 Kb R 5'ACACAGGTGA ATCATGTGGAAT; RBM33+10 Kb F 5'GAACTCAGC CTCTGTGCTGT, RBM33+10 Kb R 5'ACAATGTGA TGAGGGCTGGG; RBM33+14.2 Kb F 5'TCCTCTCTG CCAGTCTGTGA, RBM33+14.2 Kb R 5'GAAGGACT GGCATCTGGCAT; RBM33+20 Kb F 5'CCATGATT TGGAAAAGTGTGACGA, RBM33+20 Kb R 5'TGTGA CATGCTAAAAAGTTAGAGAC; $\mathrm{CLCN} 3+4 \mathrm{~Kb} F 5^{\prime}$ ACAGCATCCCTCTTGAGGAAAA, CLCN3+4 Kb R 5' GGCCCTACAGCTTTCAGTAGA; CLCN3+9.3 Kb F 5' ACGTTCTACAATGGCAGACAGA, CLCN3+9.3 Kb R 5'GCTTCGCTGACCCACTTACT; CLCN3+20 Kb F 5' ACAGGAAGGGAGAGCCAAGA, CLCN3+20 Kb R 5' AGCTGCACTCATGAACAGTCA; 18S F 5'CGCCGCT AGAGGTGAAATTCT, $18 \mathrm{~S}$ R 5'CATTCTTGGCAAAT GCTTTCG.

\section{Data availability}

Raw data have been deposited into GEO with accession number: GSE57116.

\section{Additional files}

Additional file 1: Related to Figure 1: Schematic representation of biological samples for 4sUDRB-seq. HeLa cells were either not treated (NT) or treated with DRB for 3 hours and harvested either 4 or 8 min following DRB removal. In both cases, the cells were pulsed with $4 \mathrm{sU}$ for the last $8 \mathrm{~min}$ before being harvested.

Additional file 2: Related to Figure 2: Correlation between biological repeats. Pearson correlation between the average signals over introns for all genes longer than 10Kb. The specific treatment (NT, $0 \mathrm{~min}, 4 \mathrm{~min}, 8 \mathrm{~min}$ ) and the sample number $(1,2,3,4)$ are indicated for each repeat sample.
Additional file 3: Related to Figure 3: Confidence interval distribution. Confidence interval distribution for all genes for which elongation boundaries were successfully detected by our algorithm in the 4 and 8 min samples. Genes left to the red dotted line were taken for further analysis as described in the text.

Additional file 4: Related to Figure 4: List of elongation rates and their confidence intervals. Only genes with confidence intervals better than $\pm 0.5 \mathrm{~Kb}$ were included.

Additional file 5: Related to Figure 5: Validation of transcription elongation rates. (A, B) qRT-PCR analysis of RBM33 and CLCN3 premRNA in HeLa cells, without DRB treatment (NT) and at the indicated time points after DRB removal. To simulate the experimental conditions of the DRB-seq experiment, $4 \mathrm{~s} U$ was added to all cultures $8 \mathrm{~min}$ before harvesting, although subsequent biotinylation and purification were not performed because the use of intronic primers in the GRT-PCR procedure already selects for pre-mRNA. All values were normalized to $18 \mathrm{~S}$ RNA in the same sample. Bars indicate averages of data from duplicate $\mathrm{QPCR}$ reactions; error bars represent standard deviation. (A) and (B) are derived from two independent experiments, using different qPCR primer pairs.

Additional file 6: Related to Figure 6: List of relative transcription initiation frequencies. Relative initiation frequencies were calculated by averaging slope values of four biological repeats of the 8 min samples. Outliers were excluded.

Additional file 7: Related to Figure 7: Correlation of relative initiation frequencies calculated either with or without inclusion of the first $2 \mathrm{~Kb}$ of genes. Scatter plot of relative initiation frequencies calculated for full transcripts ( $x$-axis) vs. relative initiation frequencies calculated after excluding the first $2 \mathrm{~Kb}$ of each transcript ( $\mathrm{y}$-axis).

\section{Competing interests}

The authors declare that they have no competing interests.

\section{Authors' contributions}

GF, YV, IA and MO conceived the study and designed the experiments. GF performed the experiments. $\mathrm{YV}$ analyzed the data. SB and SG generated the libraries for sequencing and performed the sequencing. GF, YV and MO wrote the paper. All authors have read and approved the manuscript for publication.

\section{Acknowledgements}

We thank Michal Rabani, Eitan Yaffe, llya Soifer and Dror Hollander for helpful discussions and Minho Chae and W. Lee Kraus for sharing with us the implementation of the Gro-HMM. This work was supported in part by a student-initiated project grant from the Kahn Center for Systems Biology, grant 293438 (RUBICAN) from the European Research Council, the Dr. Miriam and Sheldon G. Adelson Medical Research Foundation, and the Lower Saxony-Israeli Association. MO is incumbent of the Andre Lwoff chair in molecular biology.

\section{Author details}

'Department of Molecular Cell Biology, Weizmann Institute of Science, Rehovot 76100, Israel. ${ }^{2}$ Department of Molecular Genetics, Weizmann Institute of Science, Rehovot 76100, Israel. ${ }^{3}$ The Israel National Center for Personalized Medicine (INCPM), Weizmann Institute of Science, Rehovot 76100 , Israel. ${ }^{4}$ Department of Immunology, Weizmann Institute of Science, Rehovot 76100, Israel.

Received: 10 February 2014 Accepted: 9 May 2014 Published: 9 May 2014

\section{References}

1. Rasmussen $E B$, Lis JT: In vivo transcriptional pausing and cap formation on three Drosophila heat shock genes. Proc Natl Acad Sci U S A 1993, 90:7923-7927.

2. Proudfoot NJ, Furger A, Dye MJ: Integrating mRNA processing with transcription. Cell 2002, 108:501-512.

3. Neugebauer KM: On the importance of being co-transcriptional. J Cell Sci 2002, 115:3865-3871. 
4. Bentley DL: Rules of engagement: co-transcriptional recruitment of pre-mRNA processing factors. Curr Opin Cell Biol 2005, 17:251-256.

5. Hirose $Y$, Manley JL: RNA polymerase II is an essential mRNA polyadenylation factor. Nature 1998, 395:93-96.

6. Hirose Y, Tacke R, Manley JL: Phosphorylated RNA polymerase II stimulates pre-mRNA splicing. Genes Dev 1999, 13:1234-1239.

7. de la Mata M, Alonso CR, Kadener S, Fededa JP, Blaustein M, Pelisch F, Cramer P, Bentley D, Kornblihtt AR: A slow RNA polymerase II affects alternative splicing in vivo. Mol Cell 2003, 12:525-532.

8. Fuda NJ, Ardehali MB, Lis JT: Defining mechanisms that regulate RNA polymerase II transcription in vivo. Nature 2009, 461:186-192.

9. Core LJ, Waterfall JJ, Lis JT: Nascent RNA sequencing reveals widespread pausing and divergent initiation at human promoters. Science 2008, 322:1845-1848.

10. Guenther MG, Levine SS, Boyer LA, Jaenisch R, Young RA: A chromatin landmark and transcription initiation at most promoters in human cells. Cell 2007, 130:77-88

11. Muse GW, Gilchrist DA, Nechaev S, Shah R, Parker JS, Grissom SF, Zeitlinger J, Adelman K: RNA polymerase is poised for activation across the genome. Nat Genet 2007, 39:1507-1511.

12. Nechaev S, Fargo DC, dos Santos G, Liu L, Gao Y, Adelman K: Global analysis of short RNAs reveals widespread promoter-proximal stalling and arrest of Pol II in Drosophila. Science 2010, 327:335-338.

13. Bentley $\mathrm{DL}$, Groudine $\mathrm{M}$ : A block to elongation is largely responsible for decreased transcription of c-myc in differentiated HL60 cells. Nature 1986, 321:702-706.

14. Rougvie AE, Lis JT: The RNA polymerase II molecule at the $5^{\prime}$ end of the uninduced hsp70 gene of D. melanogaster is transcriptionally engaged. Cell 1988, 54:795-804.

15. Zeitlinger J, Stark A, Kellis M, Hong JW, Nechaev S, Adelman K, Levine M, Young RA: RNA polymerase stalling at developmental control genes in the Drosophila melanogaster embryo. Nat Genet 2007, 39:1512-1516.

16. Schor IE, Rascovan N, Pelisch F, Allo M, Kornblihtt AR: Neuronal cell depolarization induces intragenic chromatin modifications affecting NCAM alternative splicing. Proc Natl Acad Sci U S A 2009, 106:4325-4330.

17. Ip JY, Schmidt D, Pan Q, Ramani AK, Fraser AG, Odom DT, Blencowe BJ: Global impact of RNA polymerase II elongation inhibition on alternative splicing regulation. Genome Res 2011, 21:390-401.

18. Kwak H, Fuda NJ, Core LJ, Lis JT: Precise maps of RNA polymerase reveal how promoters direct initiation and pausing. Science 2013, 339:950-953.

19. Maunakea AK, Chepelev I, Cui K, Zhao K: Intragenic DNA methylation modulates alternative splicing by recruiting MeCP2 to promote exon recognition. Cell Res 2013, 23:1256-1269.

20. Yin H, Sweeney S, Raha D, Snyder M, Lin H: A high-resolution wholegenome map of key chromatin modifications in the adult Drosophila melanogaster. PLoS Genet 2011, 7:e1002380

21. Pinto PA, Henriques T, Freitas MO, Martins T, Domingues RG, Wyrzykowska PS, Coelho PA, Carmo AM, Sunkel CE, Proudfoot NJ, Moreira A: RNA polymerase II kinetics in polo polyadenylation signal selection. EMBO J 2011, 30:2431-2444.

22. Lin C, Smith ER, Takahashi H, Lai KC, Martin-Brown S, Florens L, Washburn MP, Conaway JW, Conaway RC, Shilatifard A: AFF4, a component of the ELL/P-TEFb elongation complex and a shared subunit of MLL chimeras, can link transcription elongation to leukemia. Mol Cell 2010, 37:429-437.

23. Lin CY, Loven J, Rahl PB, Paranal RM, Burge CB, Bradner JE, Lee TI, Young RA: Transcriptional amplification in tumor cells with elevated c-Myc. Cell 2012, 151:56-67.

24. Marazzi I, Ho JS, Kim J, Manicassamy B, Dewell S, Albrecht RA, Seibert CW, Schaefer U, Jeffrey KL, Prinjha RK, Lee K, Garcia-Sastre A, Roeder RG, Tarakhovsky A: Suppression of the antiviral response by an influenza histone mimic. Nature 2012, 483:428-433.

25. Sobhian B, Laguette N, Yatim A, Nakamura M, Levy Y, Kiernan R, Benkirane M: HIV-1 Tat assembles a multifunctional transcription elongation complex and stably associates with the 7SK snRNP. Mol Cell 2010, 38:439-451.

26. He N, Liu M, Hsu J, Xue Y, Chou S, Burlingame A, Krogan NJ, Alber T, Zhou Q: HIV-1 Tat and host AFF4 recruit two transcription elongation factors into a bifunctional complex for coordinated activation of HIV-1 transcription. Mol Cell 2010, 38:428-438.

27. He N, Zhou Q: New insights into the control of HIV-1 transcription: when Tat meets the 7SK snRNP and super elongation complex (SEC). J Neuroimmune Pharmacol 2011, 6:260-268.
28. Ardehali MB, Lis JT: Tracking rates of transcription and splicing in vivo. Nat Struct Mol Biol 2009, 16:1123-1124.

29. Danko CG, Hah N, Luo X, Martins AL, Core L, Lis JT, Siepel A, Kraus WL: Signaling pathways differentially affect RNA polymerase II initiation, pausing, and elongation rate in cells. Mol Cell 2013, 50:212-222.

30. Singh J, Padgett RA: Rates of in situ transcription and splicing in large human genes. Nat Struct Mol Biol 2009, 16:1128-1133.

31. Kim YJ, Greer CB, Cecchini KR, Harris LN, Tuck DP, Kim TH: HDAC inhibitors induce transcriptional repression of high copy number genes in breast cancer through elongation blockade. Oncogene 2013, 32:2828-2835.

32. Saunders A, Core LJ, Sutcliffe C, Lis JT, Ashe HL: Extensive polymerase pausing during Drosophila axis patterning enables high-level and pliable transcription. Genes Dev 2013, 27:1146-1158.

33. Luo X, Chae M, Krishnakumar R, Danko CG, Kraus WL: Dynamic reorganization of the $\mathrm{AC} 16$ cardiomyocyte transcriptome in response to TNFalpha signaling revealed by integrated genomic analyses. BMC Genomics 2014, 15:155.

34. Dolken L, Ruzsics Z, Radle B, Friedel CC, Zimmer R, Mages J, Hoffmann R, Dickinson P, Forster T, Ghazal P, Koszinowski UH: High-resolution gene expression profiling for simultaneous kinetic parameter analysis of RNA synthesis and decay. RNA 2008, 14:1959-1972.

35. Windhager L, Bonfert T, Burger K, Ruzsics Z, Krebs S, Kaufmann S, Malterer G, L'Hernault A, Schilhabel M, Schreiber S, Rosenstiel P, Zimmer R, Eick D, Friedel CC, Dolken L: Ultrashort and progressive 4sU-tagging reveals key characteristics of RNA processing at nucleotide resolution. Genome Res 2012, 22:2031-2042.

36. Zhou HL, Hinman MN, Barron VA, Geng C, Zhou G, Luo G, Siegel RE, Lou H: Hu proteins regulate alternative splicing by inducing localized histone hyperacetylation in an RNA-dependent manner. Proc Natl Acad Sci U S A 2011, 108:E627-E635

37. Fuchs G, Shema E, Vesterman R, Kotler E, Wolchinsky Z, Wilder S, Golomb L, Pribluda A, Zhang F, Haj-Yahya M, Feldmesser E, Brik A, Yu X, Hanna J, Aberdam $D$, Domany $E$, Oren M: RNF20 and USP44 regulate stem cell differentiation by modulating H2B monoubiquitylation. Mol Cell 2012, 46:662-673.

38. Weinberger L, Voichek Y, Tirosh I, Hornung G, Amit I, Barkai N: Expression noise and acetylation profiles distinguish HDAC functions. Mol Cell 2012 47:193-202.

39. Palangat M, Larson DR: Complexity of RNA polymerase II elongation dynamics. Biochim Biophys Acta 1819, 2012:667-672.

40. Ehrensberger AH, Kelly GP, Svejstrup JQ: Mechanistic interpretation of promoter-proximal peaks and RNAPII density maps. Cell 2013, 154:713-715.

41. Veloso A, Kirkconnell KS, Magnuson B, Biewen B, Paulsen MT, Wilson TE, Ljungman M: Rate of elongation by RNA polymerase II is associated with specific gene features and epigenetic modifications. Genome Res 2014, [Epub ahead of print].

42. Rabani M, Levin JZ, Fan L, Adiconis X, Raychowdhury R, Garber M, Gnirke A, Nusbaum C, Hacohen N, Friedman N, Amit I, Regev A: Metabolic labeling of RNA uncovers principles of RNA production and degradation dynamics in mammalian cells. Nat Biotechnol 2011, 29:436-442.

43. Langmead B, Trapnell C, Pop M, Salzberg SL: Ultrafast and memoryefficient alignment of short DNA sequences to the human genome. Genome Biol 2009, 10:R25.

44. Raney BJ, Cline MS, Rosenbloom KR, Dreszer TR, Learned K, Barber GP, Meyer LR, Sloan CA, Malladi VS, Roskin KM, Suh BB, Hinrichs AS, Clawson H, Zweig AS, Kirkup V, Fujita PA, Rhead B, Smith KE, Pohl A, Kuhn RM, Karolchik D, Haussler D, Kent WJ: ENCODE whole-genome data in the UCSC genome browser (2011 update). Nucleic Acids Res 2011, 39:D871-D875.

doi:10.1186/gb-2014-15-5-r69

Cite this article as: Fuchs et al:: 4sUDRB-seq: measuring genomewide transcriptional elongation rates and initiation frequencies within cells. Genome Biology 2014 15:R69. 